\title{
Diosgenin, a Plant-Derived Sapogenin, Enhances Regulatory T-Cell Immunity in the Intestine of Mice with Food Allergy
}

\author{
Chung-Hsiung Huang, ${ }^{\dagger}$ Der-Zen Liu, ${ }^{\ddagger}$ and Tong-Rong Jan*, ${ }^{*}$ \\ Department and Graduate Institute of Veterinary Medicine, National Taiwan University, Taipei, Taiwan 106, Republic of China, and \\ Graduate Institute of Biomedical Materials and Engineering, Taipei Medical University, Taipei, Taiwan 110, Republic of China
}

Received October 28, 2009

\begin{abstract}
It was hypothesized that the suppressive effect of diosgenin (1) on the intestinal T helper (Th)2 responses is associated with an enhancement of the regulatory T-cell immunity. Ovalbumin (OVA)-sensitized BALB/c mice were gavaged daily with 1 and received repeatedly oral OVA challenges to induce intestinal allergic responses. The expression of Th2- and Treg-related cytokines and transcription factors was examined by immunohistochemical staining and RTPCR. Administration of $\mathbf{1}$ markedly attenuated the intestinal expression of interleukin (IL)-4 and GATA3. In addition, administration of 1 reversed the diminished density of intestinal Foxp3 ${ }^{+}$cells induced by OVA oral challenges and enhanced the expression of IL-10 by Foxp $3^{+}$cells markedly. These results suggest that the suppressive effect of 1 on allergen-induced intestinal Th2 responses is closely associated with an up-regulation of the regulatory T-cell immunity in the inflammatory site.
\end{abstract}

Dioscorea opposita Thunb. (Dioscoreaceae), also known as the Chinese yam, has been used as a tonic herb and food supplement in several Asian countries for centuries. In general, D. opposita is considered as having a number of beneficial effects, such as antidiarrhea activity and the improvement of the function of the immune and gastrointestinal systems. ${ }^{1-5}$ Although the medicinal and dietary use of $D$. opposita has a long history, limited evidence is available pertaining to its claimed effects on the immune system.

Diosgenin (1) is the major steroidal sapogenin contained in the rhizomes of D. opposita and several other plants. ${ }^{1}$ Recent studies have indicated that $\mathbf{1}$ may be an active constituent contributing to some of the biological effects induced by $D$. opposita, including anti-inflammatory, antitumor, estrogenic, hypocholesterolemic, and immunomodulatory activities. We reported previously that administration of $\mathbf{1}$ attenuated the serum level of $\mathrm{IgE}$, but enhanced $\operatorname{IgG}_{2 \mathrm{a}}$ in ovalbumin (OVA)-sensitized mice. In parallel with the profile on antibody production, $\mathbf{1}$ demonstrated a suppressive effect on the expression of IL-4, but enhanced IFN- $\gamma$ expression by splenocytes. ${ }^{6,7}$ These results indicate a Th1 polarization of the Th1/ Th2 immunobalance in mice treated with 1 . Recently, our group further showed that allergen-induced intestinal inflammation was significantly attenuated by administration of $\mathbf{1}$ in a murine model of food allergy. ${ }^{7}$ Collectively, these results provide evidence to show the potential of $\mathbf{1}$ as an immunomodulatory and antiallergic phytochemical. However, the underlying immunological mechanisms for antiallergic effects mediated by $\mathbf{1}$ remain mostly unclear.

Food allergy is an increasingly prevalent atopic disorder in developed countries. ${ }^{8-10}$ The major identified food allergens are water-soluble glycoproteins, such as ovalbumin (OVA) in eggs. ${ }^{11,12}$ Food allergy is defined as an adverse immune reactivity to dietary antigens with symptoms ranging from gastrointestinal discomfort to anaphylactic shock and even death. ${ }^{13,14}$ The majority of recognized food allergy is IgE-mediated and is more prevalent in atopic individuals. Th2 cells express a battery of cytokines, including IL-4, -5, -6, and -13, which promote IgE class switching and allergic reactions. ${ }^{15}$ The increase in prevalence and potential fatality of food allergy has stimulated increasing efforts to find effective therapeutic and preventive measures. ${ }^{16}$ However, the management of food allergy relies on avoidance of allergens.

* To whom correspondence should be addressed. Tel: +886-2-33661287. Fax: +886-2-23661475. E-mail: tonyjan@ntu.edu.tw.

National Taiwan University.;

$\leftarrow$ Taipei Medical University.
Pharmacotherapy is used primarily for the relief of hypersensitivity symptoms. ${ }^{12}$ New therapeutic and preventive strategies are needed for the management of this disorder.

The gut has a life-long exposure to a variety of dietary and enteric bacterial antigens. In spite of the exposure, the gastrointestinal immune system remains insensitive to almost all of the exposed antigens primarily due to the immunosuppressive function of regulatory $\mathrm{T}$ (Treg) cells. ${ }^{17}$ Treg cells are critical for maintaining the balance between immune tolerance and allergic responses. ${ }^{18-20}$ Based on the ontogeny, Treg cells in the gut can be categorized into three major subsets, including natural Treg cells, induced Treg cells, and unconventional Treg cells. ${ }^{21}$ The transcription factor forkhead box P3 (Foxp3) is crucial for the development of natural Treg cells, which is also the most universal Treg marker widely used. $^{22,23}$ The immunosuppressive mechanisms of Treg cells include cytokine-dependent and -independent pathways. IL-10, TGF- $\beta$, and T-lymphocyte-associated molecule- 4 are the major cytokines and molecule to suppress immune responses in the cytokine-dependent and -independent pathways, respectively. Compelling evidence indicates that IL-10 and TGF- $\beta$ are crucial for maintaining immune tolerance and attenuating allergic responses. ${ }^{24-28}$

On the basis of the pivotal role of Treg cells in maintaining the intestinal immune homeostasis, it is hypothesized that the antiallergic effect of $\mathbf{1}$ may be associated with the up-regulation of regulatory T-cell immunity. To test this hypothesis, we examined the influence of the administration of $\mathbf{1}$ on the profile of the Th1/ Th2 immunobalance and the number and functionality of Foxp $3^{+}$ cells in the intestine using a murine model of food allergy induced by OVA.

\section{Results and Discussion}

Diosgenin (1) Administration Attenuated Allergen-Induced Th2 Immune Responses in the Intestine. It has been well established that T-cells play a pivotal role in the pathogenesis of food allergy and the maintenance of intestinal immune homeostasis. ${ }^{21,29,30}$ To elucidate the underlying immunological mechanisms for 1-mediated antiallergic effects, we investigated whether diosgenin affects the functionality of T-cells in the intestine, which is one of the major inflammatory sites associated with food allergy. The duodenal sections from the OVA and VH control groups, which have been shown to exhibit marked inflammatory responses, ${ }^{7}$ showed red signals around the blue nuclei, indicating IL- $4^{+}$cells (Figure 1A). The number of IL- $4^{+}$cells was increased 

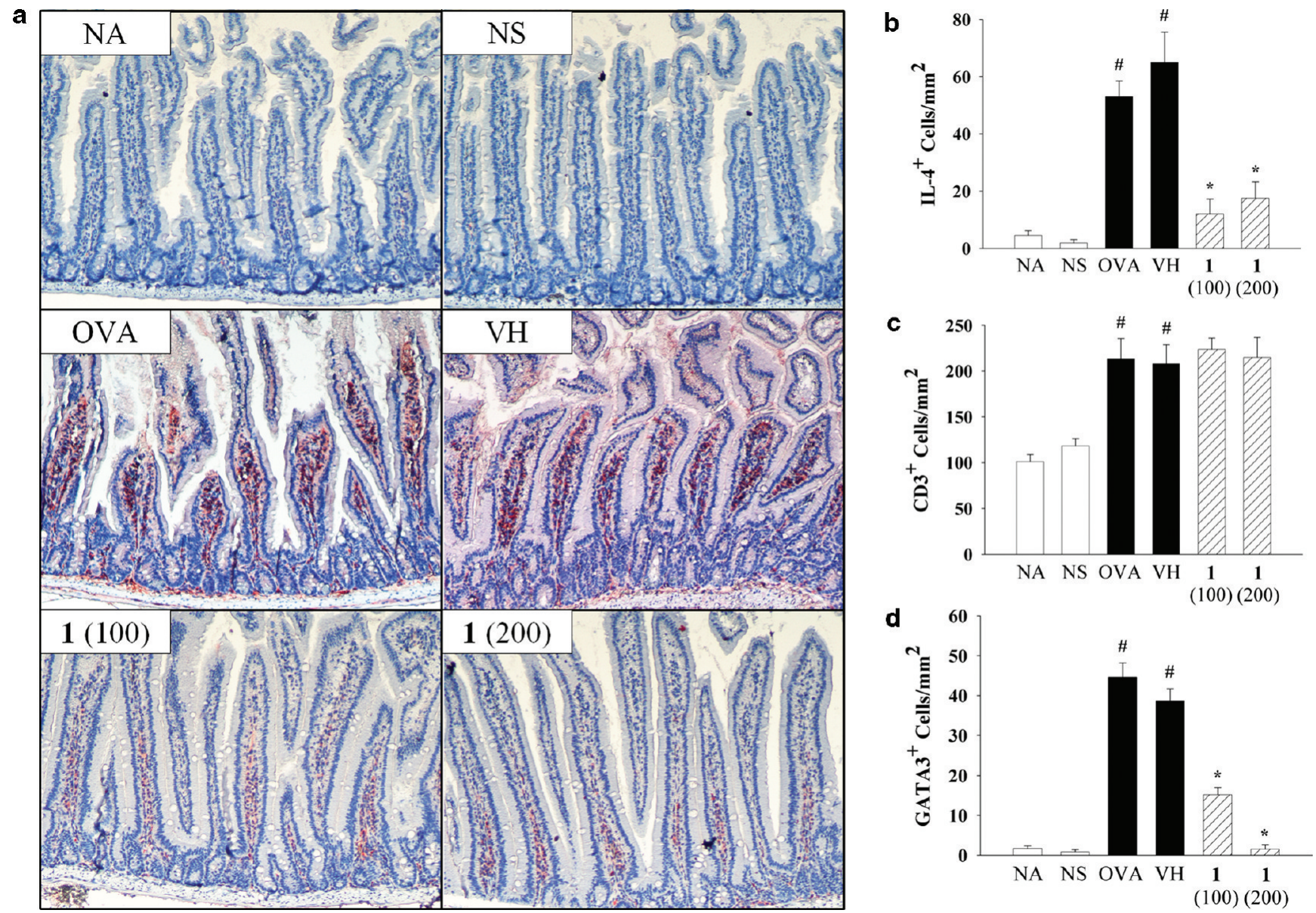

Figure 1. Immunohistochemical analysis of IL-4, CD3, and GATA3 in the duodenum. (A) Representative sections stained for IL-4 are shown (original magnification, $\times 100)$. Cells with red signals around the blue nuclei indicate IL-4 ${ }^{+}$cells. The positive cells of $(B)$ IL-4, $(C)$ CD3, and (D) GATA3 are quantified and expressed as means \pm SEM $(n=3-6)\left({ }^{\#} p<0.05\right.$ compared to the NS group; $* p<0.05$ compared to the $\mathrm{VH}$ group; results are representative of three independent experiments).

markedly in the OVA group compared to the NS group (Figure $1 \mathrm{~A}, \mathrm{~B})$, confirming the activation of Th2 cells in response to OVA challenges in the employed model. Allergic diarrhea and serum IgE were also confirmed in the OVA group (data not shown). The number of $\mathrm{IL}_{-} 4^{+}$cells in the $\mathrm{VH}$ group was similar to that of the OVA group, demonstrating that the VH per se did not affect the cytokine response (Figure 1B). The IL-4 response induced by OVA challenges was suppressed significantly by both doses of $\mathbf{1}$ used (Figure 1B; VH vs 1 of 100 and $200 \mathrm{mg}$ ). To address the possibility that the detected IL-4 changes may be due to altered infiltration of T-cells, the presence of $\mathrm{CD}^{+}$cells in the duodenum was examined. This possibility was ruled out, as a comparable number of $\mathrm{CD}^{+}$cells was observed between the 1- and VH-treated groups (Figure 1C). However, a greater number of $\mathrm{CD}^{+}$cells was detected in the OVA group compared to the NS group (Figure 1C), confirming the involvement of T-cells in allergic inflammation. In addition to cytokine, the expression of GATA3 was examined, since this is a key transcription factor involved in the development of Th2 cells. The number of GATA $3^{+}$cells was increased by OVA challenge (Figure 1D; NS vs OVA), which was suppressed significantly by administration of $\mathbf{1}$ (Figure 1D; VH vs $\mathbf{1}$ of 100 and $200 \mathrm{mg}$ ). These results demonstrate the attenuation by $\mathbf{1}$ of Th2 responses in the inflammatory site and suggest that the effect may be attributed to the suppression of Th2 cell development.

Diosgenin (1) Administration Reverses Allergen-Induced Diminishment of Foxp3 ${ }^{+}$Cells and Enhances IL-10 Expression by Foxp3 $^{+}$Cells. Previous studies have shown that the regulatory T-cell immunity is critical for counteracting aberrant Th2type immune responses and that the level of splenic Foxp3 mRNA expression is diminished in a murine model of food allergy. ${ }^{31,32}$
Table 1. Immunohistochemical Staining of Foxp $3^{+}$, IL- $10^{+}$, and TGF- $\beta^{+}$Cells in the Duodenum:

\begin{tabular}{lrrcccc}
\hline & \multicolumn{6}{c}{ number of positive cells } \\
\cline { 2 - 7 } & \multicolumn{1}{c}{ NA } & \multicolumn{1}{c}{ NS } & \multicolumn{1}{c}{ OVA } & VH & $\mathbf{1}(100)$ & $\mathbf{1}(200)$ \\
\hline Foxp3 $^{+}$ & $62 \pm 9$ & $49 \pm 4$ & $19 \pm 11^{b}$ & $16 \pm 14$ & $59 \pm 9^{c}$ & $86 \pm 11^{c}$ \\
IL-10 $^{+}$ & $5 \pm 3$ & $9 \pm 3$ & $21 \pm 1^{b}$ & $26 \pm 3$ & $50 \pm 8^{c}$ & $49 \pm 7^{c}$ \\
TGF- $^{+}$ & $34 \pm 3$ & $34 \pm 3$ & $41 \pm 3$ & $32 \pm 2$ & $35 \pm 2$ & $33 \pm 4$ \\
Foxp3 $^{+}$IL-10 $^{+}$ & $1 \pm 1$ & $2 \pm 1$ & $4 \pm 1$ & $4 \pm 2$ & $21 \pm 4^{c}$ & $22 \pm 4^{c}$ \\
\hline
\end{tabular}

${ }^{a}$ The number of positive cells was quantified using Image-Pro Plus 5.1 morphometric software, except for Foxp $3^{+} \mathrm{IL}-10^{+}$cells, which were counted manually. Data are expressed as means \pm SEM $(n=3-6)$. Results are representative of three independent experiments. ${ }^{b} p<0.05$ compared to the NS group. ${ }^{c} p<0.05$ compared to the VH group.

The pivotal role of Treg cells in regulating intestinal allergic responses has been illustrated recently using Peyer patch-null mice. ${ }^{33}$ To address whether administration of $\mathbf{1}$ influences the intestinal regulatory T-cell immunity, the presence of Foxp $3^{+}$cells and the expression of IL-10 and TGF- $\beta$, two major inhibitory cytokines known to be secreted by Treg cells, were examined. ${ }^{24-27}$ A decreased number of Foxp $3^{+}$cells was detected in the OVA group compared to the NS group (Table 1), confirming the association of Treg cells with the pathophysiology of food allergy. Interestingly, an increased number of IL-10 $10^{+}$cells in the OVA group was observed (Table 1). The IHC results were confirmed by RTPCR using total RNA extracts of the duodenum. The levels of Foxp3 and IL-10 mRNA expression in the OVA group were significantly attenuated and enhanced, respectively, compared to the NS group (Figure 2A,B,C). These results are inconsistent with the notion that IL-10 is expressed by Treg cells, which can be 

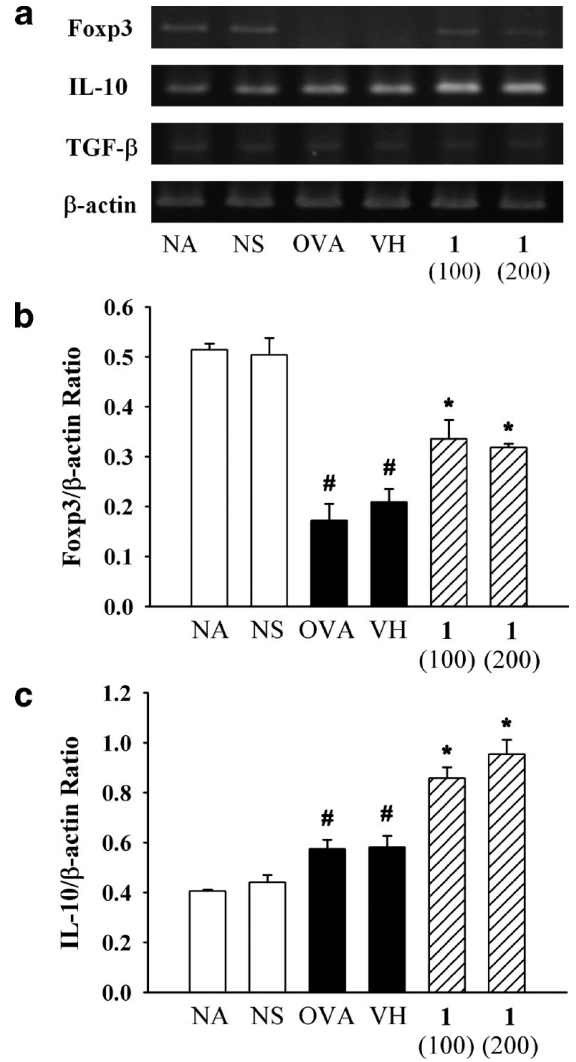

Figure 2. mRNA expression of Foxp3, IL-10, and $\beta$-actin in the duodenum. (A) Representative photos of RT-PCR products are shown. The expression levels of (B) Foxp3 and (C) IL-10 are quantified and expressed as means $\pm \operatorname{SEM}(n=3-6)\left({ }^{\#} p<0.05\right.$ compared to the NS group; $* p<0.05$ compared to the VH group; results are representative of three independent experiments). marked as Foxp $3^{+}$. To address this issue and elucidate the source of IL-10, doubling staining for both Foxp3 and IL-10 was performed. The number of Foxp $3^{+} \mathrm{IL}-10^{+}$cells in the OVA group is low and comparable to the NS group (Table 1). These results indicate that Foxp $3^{+}$cells are not the major source responsible for the increased IL-10 expression in the OVA group. Alternatively, as Th2 cells can produce IL-10, ${ }^{34}$ and the results showed an increased $\mathrm{CD}^{+}$cell infiltration and IL-4 expression in the duodenum of OVA-challenged mice (Figure 1), it is postulated that the infiltrated $\mathrm{CD}^{+}$cells, likely $\mathrm{Th} 2$ cells, in the duodenum may account for the increased IL-10 expression.

Diosgenin (1) administration increased both the number of Foxp $^{+}$and IL- $10^{+}$cells compared to the VH control group (Table 1). Results from IHC doubling staining further showed a significant increase in the number of Foxp $3^{+} \mathrm{IL}-10^{+}$cells in 1-treated groups compared to the VH group (Table 1 and Figure 3). The IHC results on Foxp3 and IL-10 were confirmed by RT-PCR analysis (Figure $2 \mathrm{~B}, \mathrm{C})$. In contrast to the marked changes in IL-10 expression, the number of TGF- $\beta^{+}$cells was comparable among the different groups (Table 1), showing that IL-10, but not TGF- $\beta$, may contribute to the antiallergic effect of $\mathbf{1}$. Of relevance to the present findings, the activity of IL-10 as a regulatory cytokine to inhibit the development of allergic diarrhea has been previously reported in the OVA model employed. ${ }^{33,35,36}$ To the best of our knowledge, the present studies provide the first evidence to show that intake of 1 up-regulates regulatory T-cell immunity, which may account for the antiallergic effect of $\mathbf{1}$. As $\mathbf{1}$ is the main sapogenin contained in the rhizomes of $D$. opposita, ingestion of the Chinese yam may be beneficial for the prevention of allergy. It is presently unclear how 1 up-regulates the regulatory T-cell immunity. Our preliminary results suggest that $\mathbf{1}$ possesses a prebiotic-like activity on certain lactic acid bacteria (unpublished data), which might be a potential mechanism responsible for the antiallergic effect of $\mathbf{1}$.

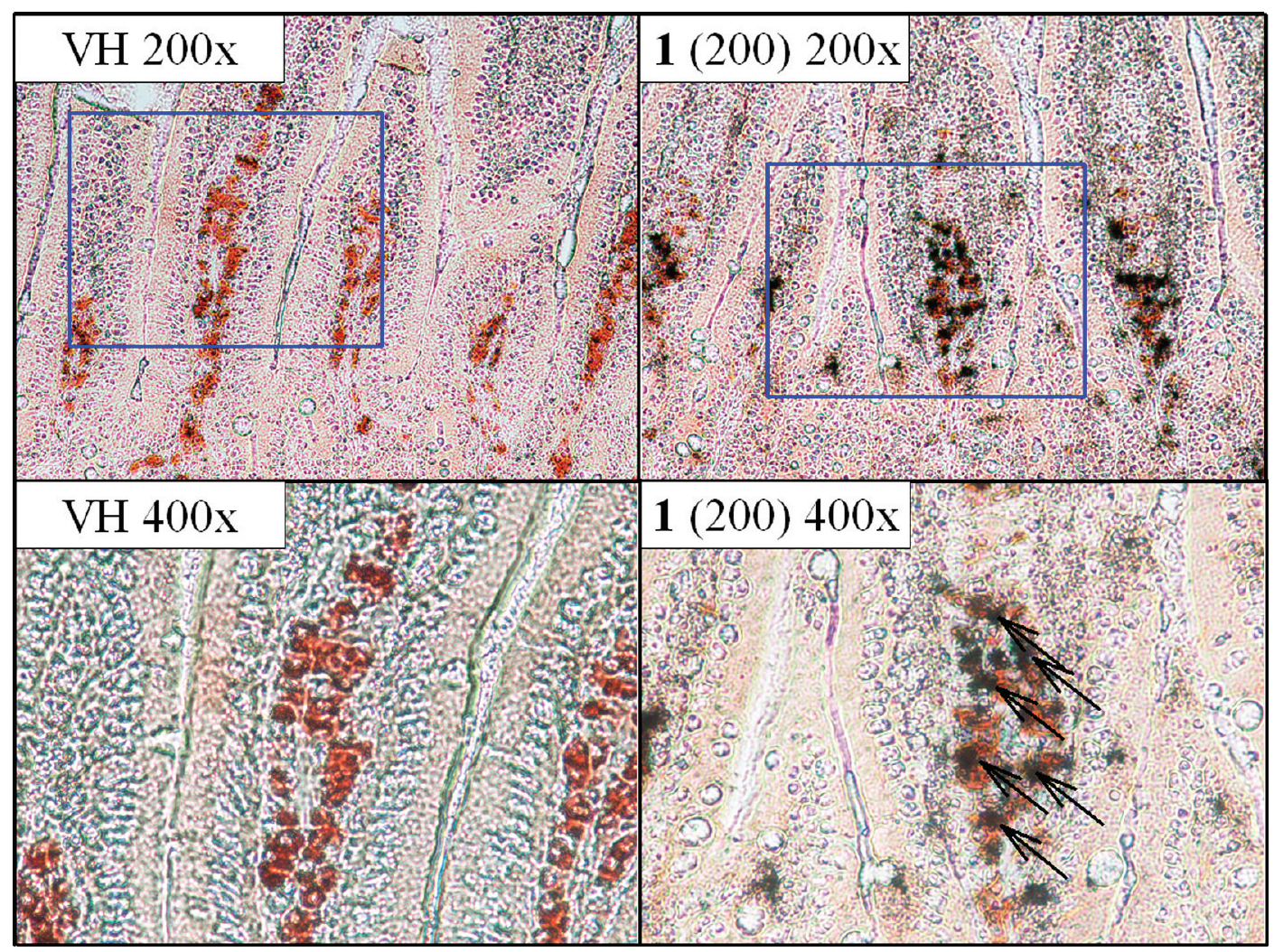

Figure 3. IL-10-secreting Foxp $3^{+}$cells in the duodenum. Representative sections stained for both IL-10 and Foxp3 are shown. Arrows indicate Foxp $3^{+} \mathrm{IL}-10^{+}$cells. Quantitative data are shown in Table 1. 


\section{Experimental Section}

Mice, Reagents, and Antibodies. Male BALB/c mice (4 to 5 weeks old) were obtained from the Animal Breeding Center of the National Taiwan University Hospital (Taipei, Taiwan). On arrival, mice were transferred randomly to plastic cages containing a sawdust bedding and quarantined for 1 week. Mice were housed in a temperature- $(23 \pm 2$ $\left.{ }^{\circ} \mathrm{C}\right)$, humidity- $(60 \pm 20 \%)$, and light- (12 h light/dark cycle) controlled environment. Except on the days of allergen challenge (described in the following section), food and water were supplied ad libitum.

All chemicals and reagents, including diosgenin (1, purity $\geq 99 \%)$, were purchased from Sigma Chemical (St. Louis, MO), unless otherwise stated. Reagents used for immunohistochemical (IHC) staining were purchased from BioGenex Laboratories (San Ramon, CA) and AbCam, Inc. (Cambridge, MA). Reagents and enzymes used for RT-PCR were purchased from Promega (Madison, WI).

The following purified antibodies were used for IHC staining: antiCD3 IgG (Neomarker, Fremont, CA), anti-mouse IL-4 IgG 1 , anti-mouse IFN- $\gamma \operatorname{IgG}_{1}$, anti-mouse Foxp3 $\mathrm{IgG}_{2 \mathrm{~b}}$, anti-mouse IL-10 $\mathrm{IgG}_{2 \mathrm{~b}}$ (all from BioLegend, San Diego, CA), ant-mouse GATA3 IgG (AbCam), antimouse TGF- $\beta$ IgG (ABBIOTEC, San Diego, CA), and anti-mouse T-bet IgG (Santa Cruz Biotechnology, Inc., Santa Cruz, CA). The anti-rat IgG secondary antibodies conjugated with alkaline phosphatase (AP) or horse radish peroxidase (HRP) were purchased from AbCam, Inc.

Protocol of Animal Experiments. The experimental protocol employed and dosing regimen have been reported previously. ${ }^{6}$ Briefly, the mice were divided randomly into the following groups (Figure S1, Supporting Information): naïve (NA), nonsensitized (NS), OVAsensitized and challenged (OVA), vehicle-treated, OVA-sensitized, and challenged (VH), and treated with 1, OVA-sensitized, and challenged. Diosgenin (1) was suspended in olive oil (vehicle; VH) as a fine suspension. This compound (100-200 mg/kg body weight; 1100 and $1200 ; 0.1 \mathrm{~mL} /$ mouse $)$ and $\mathrm{VH}(0.1 \mathrm{~mL} /$ mouse $)$ were administered daily into mice by oral gavage throughout the experiment. Except for the mice in the NA and NS groups, each mouse was sensitized with OVA by intraperitoneal injection using $0.1 \mathrm{~mL}$ of sensitization solution containing $50 \mu \mathrm{g}$ of OVA and $1 \mathrm{mg}$ of alum on day 3 and later boosted with a double dose on day 17 followed by repeated challenge with OVA by gavage every other day from day 31 to day 49 . Mice were deprived of food $3 \mathrm{~h}$ before administration of $\mathbf{1}$ on the days of challenge and were challenged $1 \mathrm{~h}$ after administration of this compound. The mice were euthanized $3 \mathrm{~h}$ after the last OVA challenge, and the duodenal tissues were isolated for further experiments. The animal experiments were approved by the Institutional Animal Care and Use Committee of the National Taiwan University.

Immunohistochemistry. The duodenal tissues (1.5 to $4.5 \mathrm{~cm}$ toward the anal side from the pylorus) were excised followed by washing with saline and then fixed in $10 \%$ neutral buffered formalin. After $24 \mathrm{~h}$, tissue blocks were embedded in paraffin and cut into 4-5 $\mu \mathrm{m}$ thick sections on silane-coated slides. For dewaxing, slides were immersed in xylene for $5 \mathrm{~min}$ three times and then immersed sequentially in $100 \%$, $95 \%, 90 \%, 80 \%$, and $60 \%$ ethanol for 5 min each for hydration. The hydrated slides were immersed in Trilogy (Cell Marque Corporation, Rocklin, CA) at $121{ }^{\circ} \mathrm{C}$ for $15 \mathrm{~min}$ for antigen retrieval. The slides were then treated with $3 \% \mathrm{H}_{2} \mathrm{O}_{2}$ for 15 min followed by blocking with normal horse serum for $1 \mathrm{~h}$. After washing, the slides were incubated with primary antibodies at $4{ }^{\circ} \mathrm{C}$ in the dark overnight, treated with Super Enhancer reagent for $1 \mathrm{~h}$, and then incubated with Poly-HRP reagent or HRP-conjugated secondary antibody for $1 \mathrm{~h}$. For visualization, the slides were treated with the HRP substrate 3-amino-9ethylcarbazole (AEC) for $2 \mathrm{~min}$ followed by hematoxylin counterstaining for $1 \mathrm{~min}$.

For IHC double staining, AEC-treated slides were incubated with another primary antibody at $4{ }^{\circ} \mathrm{C}$ in the dark overnight followed by incubation with AP-conjugated secondary antibody for $1 \mathrm{~h}$. Finally, the slides were treated with BCIP/NBT (5-bromo-4-chloro-3-indolylphosphate/nitroblue tetrazolium, a substrate of AP) solution for 30 min for observation of a second staining without counter-staining. The slides were washed three times between each step after dewaxing. Except for the step of antigen retrieval and incubation with primary antibodies, each step was operated in the dark at room temperature.

The number of IHC-positive signals was quantified using the ImagePro Plus 5.1 morphometric program, as previously described. ${ }^{6}$ Three measurements per tissue segment and 3-6 segments per group were analyzed at $100-$ or 400 -fold magnification.
Reverse Transcription-Polymerase Chain Reaction (RT-PCR). Total RNA from the duodenal tissues of each group was extracted using TRI reagent (Sigma) following the supplier's instruction. The steadystate mRNA expression of IL-4, IFN- $\gamma$, Foxp3, L-10, and $\beta$-actin was measured by RT-PCR. All isolated RNA samples were confirmed to be free of DNA contamination, as determined by the absence of product after PCR amplification in the absence of RT. For reverse transcription, $2 \mu \mathrm{g}$ of total RNA was reverse-transcribed into cDNA by 40 units of MMLV reverse transcriptase using oligo-dT as primers. The reverse transcription proceeded at $42{ }^{\circ} \mathrm{C}$ for $15 \mathrm{~min}$ and then at $95{ }^{\circ} \mathrm{C}$ for 5 min. A PCR master mixture consisting of PCR buffer, $4 \mathrm{mM} \mathrm{MgCl}$, 6 pmol each of forward and reverse primers specific for the gene of interest, and 2.5 units of Taq DNA polymerase was added to each cDNA sample for PCR. The used PCR primers are listed in Table S1, Supporting Information. PCR products were electrophoresed in $1.5 \%$ agarose gels and visualized by ethidium bromide staining. Quantification was performed by assessing the optical density for the DNA bands (cytokine mRNA of interest) using the Alpha Imager 1200 digital imaging system (Alpha Innotech Corp, San Leandro, CA). The results are expressed as the density ratio between the gene of interest and the reference standard ( $\beta$-actin).

Statistical Analysis. Data are expressed as the mean \pm standard error of the mean (SEM) for each treatment group. Dunnett's twotailed $t$-test was used to assess the statistical difference between the NA control group, VH control group, and treatment groups $(p<0.05$ was defined as statistically significant).

Acknowledgment. This work was supported by grants NSC98-2923B-002-001-MY2 and NSC98-2320-B-002-036-MY3 from the National Science Council, Executive Yuan, Taiwan.

Supporting Information Available: Table of primers used for RTPCR analysis and figure of the protocol used for diosgenin (1) administration and OVA sensitization and challenge. This information is available free of charge via the Internet at http://pubs.acs.org.

\section{References and Notes}

(1) Choi, E. M.; Koo, S. J.; Hwang, J. K. J. Ethnopharmacol. 2004, 91, $1-6$.

(2) Attele, A. S.; Wu, J. A.; Yuan, C. S. Biochem. Pharmacol. 1999, 58, $1685-1693$

(3) Roman, I. D.; Thewles, A.; Coleman, R. Biochim. Biophys. Acta 1995, 1255, 77-81.

(4) Corbiere, C.; Liagre, B.; Bianchi, A.; Bordji, K.; Dauca, M.; Netter, P.; Beneytout, J. L. Int. J. Oncol. 2003, 22, 899-905.

(5) Hu, K.; Dong, A.; Yao, X.; Kobayashi, H.; Iwasaki, S. Planta Med. 1996, 62, 573-575.

(6) Jan, T. R.; Wey, S. P.; Kuan, C. C.; Liao, M. H.; Wu, H. Y. Planta Med. 2007, 73, 421-426.

(7) Huang, C. H.; Ku, C. Y.; Jan, T. R. Planta Med. 2009, 75, 13001305.

(8) Venter, C.; Pereira, B.; Grundy, J.; Clayton, C. B.; Arshad, S. H.; Dean, T. Pediatr. Allergy Immunol. 2006, 17, 356-363.

(9) Pereira, B.; Venter, C.; Grundy, J.; Clayton, C. B.; Arshad, S. H.; Dean, T. J. Allergy Clin. Immunol. 2005, 116, 884-892.

(10) Osterballe, M.; Hansen, T. K.; Mortz, C. G.; Host, A.; Bindslev-Jensen, C. Pediatr. Allergy Immunol. 2005, 16, 567-573.

(11) Noma, T.; Yoshizawa, I.; Aoki, K.; Yamaguchi, K.; Baba, M. Clin. Exp. Allergy 1996, 26, 1298-1307.

(12) Tiemessen, M. M.; Van Ieperen-Van Dijk, A. G.; Bruijnzeel-Koomen, C. A.; Garssen, J.; Knol, E. F.; Van Hoffen, E. J. Allergy Clin. Immunol. 2004, 113, 932-939.

(13) Sampson, H. A. J. Allergy Clin. Immunol. 2003, 111, S540-547.

(14) Sampson, H. A. J. Allergy Clin. Immunol. 2004, 113, 805-819.

(15) Kasakura, S. Rinsho. Byori. 1998, 46, 915-921.

(16) Skolnick, H. S.; Conover-Walker, M. K.; Koerner, C. B.; Sampson, H. A.; Burks, W.; Wood, R. A. J. Allergy Clin. Immunol. 2001, 107, 367-374.

(17) Chehade, M.; Mayer, L. J. Allergy Clin. Immunol. 2005, 115, 3-12.

(18) Chen, Y.; Inobe, J.; Weiner, H. L. J. Immunol. 1995, 155, 910-916.

(19) Barone, K. S.; Jain, S. L.; Michael, J. G. Cell. Immunol. 1995, 163 , 19-29.

(20) Garside, P.; Steel, M.; Liew, F. Y.; Mowat, A. M. Int. Immunol. 1995, 7, 501-504.

(21) Saurer, L.; Mueller, C. Allergy 2009, 64, 505-519.

(22) Zheng, Y.; Rudensky, A. Y. Nat. Immunol. 2007, 8, 457-462.

(23) Sakaguchi, S. Аnпи. Rev. Immunol. 2004, 22, 531-562.

(24) Beyer, K.; Castro, R.; Birnbaum, A.; Benkov, K.; Pittman, N.; Sampson, H. A. J. Allergy Clin. Immunol. 2002, 109, 707-713. 
(25) Oh, J. W.; Seroogy, C. M.; Meyer, E. H.; Akbari, O.; Berry, G.; Fathman, C. G.; Dekruyff, R. H.; Umetsu, D. T. J. Allergy Clin. Immunol. 2002, 110, 460-468.

(26) Meiler, F.; Zumkehr, J.; Klunker, S.; Ruckert, B.; Akdis, C. A.; Akdis, M. J. Exp. Med. 2008, 205, 2887-2898.

(27) Joetham, A.; Takeda, K.; Taube, C.; Miyahara, N.; Matsubara, S.; Koya, T.; Rha, Y. H.; Dakhama, A.; Gelfand, E. W. J. Immunol. 2007, $178,1433-1442$.

(28) Hansen, G.; McIntire, J. J.; Yeung, V. P.; Berry, G.; Thorbecke, G. J.; Chen, L.; DeKruyff, R. H.; Umetsu, D. T. J. Clin. Invest. 2000, 105, $61-70$.

(29) Tada, T.; Takemori, T.; Okumura, K.; Nonaka, M.; Tokuhisa, T. J. Exp. Med. 1978, 147, 446-458.

(30) Neurath, M. F.; Finotto, S.; Glimcher, L. H. Nat. Med. 2002, 8, 567573.
(31) Ganeshan, K.; Neilsen, C. V.; Hadsaitong, A.; Schleimer, R. P.; Luo, X.; Bryce, P. J. J. Allergy Clin. Immunol. 2009, 123, 231-238.

(32) Cardoso, C. R.; Teixeira, G.; Provinciatto, P. R.; Godoi, D. F.; Ferreira, B. R.; Milanezi, C. M.; Ferraz, D. B.; Rossi, M. A.; Cunha, F. Q.; Silva, J. S. Clin. Exp. Allergy 2008, 38, 338-349.

(33) Takayama, N.; Igarashi, O.; Kweon, M. N.; Kiyono, H. Clin. Immunol. 2007, 123, 199-208.

(34) Mosmann, T. R.; Schumacher, J. H.; Fiorentino, D. F.; Leverah, J.; Moore, K. W.; Bond, M. W. J. Immunol. 1990, 145, 2938-2945.

(35) Uhlig, H. H.; Coombes, J.; Mottet, C.; Izcue, A.; Thompson, C.; Fanger, A.; Tannapfel, A.; Fontenot, J. D.; Ramsdell, F.; Powrie, F. J. Immunol. 2006, 177, 5852-5860.

(36) Izcue, A.; Powrie, F. Immunology 2008, 123, 6-10.

NP900690Z 